\title{
ABNORMALITIES IN THE RECOVERY CYCLES IN SIMULATED DEMYELINATING NEUROPATHIES AND NEURONOPATHIES
}

\author{
Krustev S. ${ }^{1}$, D. Stephanova ${ }^{2}$ \\ ${ }^{1}$ Department of Medical Physics and Biophysics, Faculty of Pharmacy, Medical University of \\ Varna, ${ }^{2}$ Institute of Biophysics and Biomedical Engineering, \\ Bulgarian Academy of Sciences, Sofia
}

\begin{abstract}
The recovery cycles reflect the adaptive processes in nerve excitable structures. In order to expand our studies of the adaptive processes, these recovery cycles are compared in previously simulated by us demyelinating neuropathies such as Charcot-Marie-Tooth disease type 1A (CMT1A), chronic inflammatory demyelinating polyneuropathy (CIDP), CIDP subtypes, Guillain-Barre Syndrome (GBS), multifocal motor neuropathy (MMN) and neuronopathies such as amyotrophic lateral sclerosis (ALS) with three progressively greater degrees termed Type 1, Type 2, Type 3. Using our multi-layered model of human motor nerve fibre, the calculated recovery cycles are presented as in the simulated normal case and in the mentioned diseases. The increased superexcitability periods of the axonal excitability, recovered to the end of the investigated $100 \mathrm{~ms}$ cycles, are characteristic for the simulated demyelinating neuropathies and ALS Type 1. The abnormally increased superexcitability periods of the axonal excitability, which is not recovered to the end of the cycles, are characteristic for ALS Type 2 and Type 3. These axonal superexcitabilities lead to blockage of each applied third (testing) stimulus in the recovery cycle of ALS Type 2 and to blockage of each applied second (testing) stimulus in the recovery cycle of ALS Type 3 as a result of repetitive firing caused by the applied preceding stimulus (i. e. by the applied first (conditioning) and second (testing) stimuli in ALS Type 3 and ALS Type 2). The present study shows that the recovery cycle abnormalities in the simulated demyelinating neuropathies are quite different from those in ALS because of the different fibre electrogenesis.
\end{abstract}

Key words: recovery cycles, demyelinating neuropathies, amyotrophic lateral sclerosis, computational neuroscience

\section{INTRODUCTION}

The accommodative and adaptive processes are of biological importance in determining whether and how often axons discharge when excited by slowly changing subthreshold and suprathreshold prolonged stimuli, respectively. More recently, a non-invasive threshold tracking technique has been developed $(1,2,5)$ to measure nerve excitability properties in healthy subjects and patients with demyelinating neuropathies such as CMT1A, CIDP, subtypes CIDP, GBS, MMN (3,4,6-9,15) and motor neuron diseases such as ALS Type 1, Type 2 and Type 3 (3). The present study compares previously calculated by us recovery cycles in simulated normal case, mild systematic demyelinations (SD) [such as internodal (ISD) as specific indicators of CMT1A; paranodal (PSD) as specific indicators of CIDP; paranodal internodal (PISD) as specific indicators of CIDP subtypes], severe focal demyelinations (FD) [such as internodal (IFD) as specific indicators of GBS; paranodal

Address for correspondence:

D. Stephanova, Institute of Biophysics and Biomedical Engineering, Bulgarian Academy of Sciences,

Acad. G. Bontchev Str., Bl. 21, Sofia 1113, Bulgaria

e-mail: dsteph@shiva.bio.bas.bg
(PFD); and paranodal internodal (PIFD) as specific indicators of the MMN] (13) as well as in three ALS subtypes defined as ALS1, ALS2, and ALS3 as specific indicators of motor neuron disease ALS Type 1, ALS Type 2 and ALS Type 3, respectively $(12,14)$.

The results presented here are consistent with the interpretation that genetic or immunological factors, or both of them causing changes in the intermodal, or paranodal segments or both of them in the myelin sheath could be responsible for the adaptive abnormalities obtained in the simulated demyelinating neuropathies such as CMT1A, CIDP, subtypes CIDP, GBS, and MMN. Similarly, the results presented here are consistened with interpretation that genetic or immunological factors, or both of them causing changes in the nodal or internodal axolemma beneath the myelin sheath could be responsible for the adaptive abnormalities obtained in the simulated three ALS subtypes.

\section{MATERIAL AND METHODS}

Using our multi-layered model of human motor nerve fibre (11) without taken into account the myelin sheath aqueous 
layers, simulations of normal case, mild systematic and severe focal demyelinations each of them internodal, paranodal and paranodal internodal are displayed below. The investigated demyelinations are associated with a corresponding loss of the myelin end bulbs and myelin lamellae away from the axolemma (Fig. 1).

The characteristic parameter values defining the simulated cases are demonstrated in Table 1 . The $70 \%$ reduction

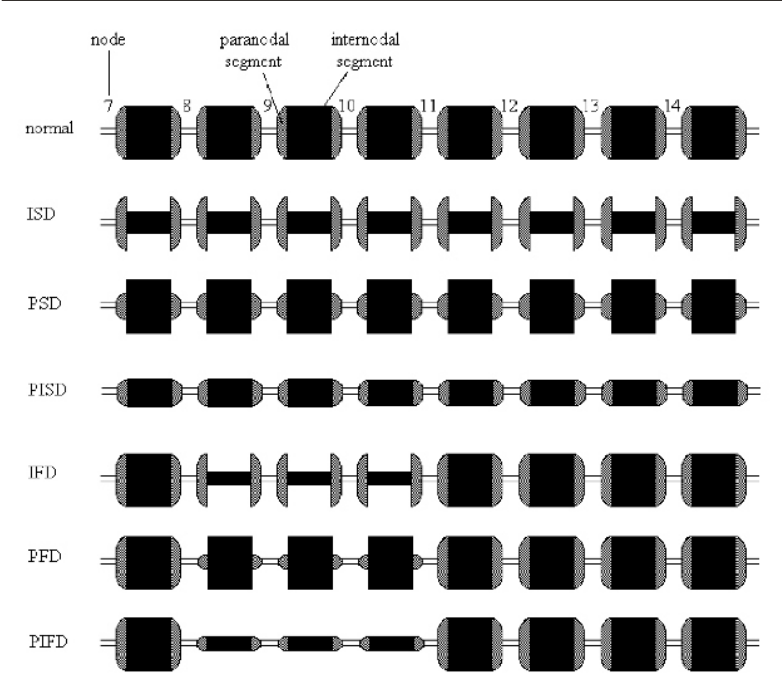

Fig. 1. Schematic diagram of human motor nerve fibres from the $7^{\text {th }}$ to the $14^{\text {th }}$ nodes in normal, systematically and focally demyelinated cases. The reduction of the myelin lamellae (defining internodal systematic demyelination, ISD), or of the paranodal seal resistance (defining paranodal systematic demyelination, PSD), or simultaneously both of them (defining paranodal internodal systematic demyelination, PISD) is uniform along the fibre length. The reduction of the same myelin parameters is restricted to only three $\left(8^{\text {th }}, 9^{\text {th }}\right.$ and $\left.10^{\text {th }}\right)$ consecutive internodes for the internodal focal demyelination (IFD), paranodal focal demyelination (PFD) and paranodal internodal focal demyelination $(P I F D)$, respectively.

Table 1. Membrane parameter values characteristic for human motor nerve fibres in normal and demyelinated cases, when the demyelinations are mild (70\%) and severe $(96 \%)$, respectively.

\begin{tabular}{||l|c|c|c|c|c||}
\hline \hline \multicolumn{2}{||l|}{} & & $\mathrm{R}_{\mathrm{my}}[\mathrm{M} \Omega]$ & $\mathrm{C}_{\mathrm{my}}[\mathrm{pF}]$ & $\mathrm{R}_{\mathrm{pn}}[\mathrm{M} \Omega]$ \\
\hline Normal & & 150 & 250 & 1.5 & 125 \\
\hline ISD & $70 \%$ & 47 & 78.3 & 4.8 & 125 \\
\hline PSD & $70 \%$ & 150 & 250 & 1.5 & 39 \\
\hline PISD & $70 \%$ & 47 & 78.3 & 4.8 & 39 \\
\hline IFD & $96 \%$ & 6 & 10 & 37.5 & 125 \\
\hline PFD & $96 \%$ & 150 & 250 & 1.5 & 5 \\
\hline PIFD & $96 \%$ & 6 & 10 & 37.5 & 5 \\
\hline \hline
\end{tabular}

$N$ (number of myelin lamellae); $R_{m y}$ (myelin resistance); $C_{m y}$ (myelin capacitance); $R_{\text {pn }}$ (paranodal seal resistance) value is not sufficient to develop a conduction block of action potential in the systematically demyelinated cases. Such demyelinations are regarded as mild. The 96\% reduction value is the first degree of the focally demyelinated subtypes for achieving the conduction block in a single internode. Such demyelinations are regarded as severe. Cases of uniform nodal and internodal axonal dysfunctions defined as ALS1, ALS2 and ALS3 were also simulated by us. Their typical parameter values are shown in Table 2.

Table 2. Membrane parameter values characteristic for human fibres in normal, ALS1, ALS2 and ALS3 cases

\begin{tabular}{||l|c|c|c|c||}
\hline \hline cases & normal & ALS1 & ALS2 & ALS3 \\
\hline $\mathrm{R}_{\mathrm{ax}}[\mathrm{M} \Omega]$ & 8 & $\underline{10}$ & 10 & 10 \\
\hline $\mathrm{R}_{\mathrm{pn}}[\mathrm{M} \Omega]$ & 125 & $\underline{145}$ & 145 & 145 \\
\hline $\mathrm{P}_{\mathrm{Na}} \cdot 10^{-9}\left[\mathrm{sm}^{3} \mathrm{~s}^{-1}\right]$ & 9 & $\underline{7}$ & 7 & 7 \\
\hline $\mathrm{P}_{\mathrm{Kf}} \cdot 10^{-9}\left[\mathrm{sm}^{3} \mathrm{~s}^{-1}\right]$ & 0.07 & $\underline{0}$ & $\underline{0.07}$ & 0.07 \\
\hline $\mathrm{P}_{\mathrm{Ks}} \cdot 10^{-9}\left[\mathrm{sm}^{3} \mathrm{~s}^{-1}\right]$ & 0.26 & $\underline{0.13}$ & $\underline{0}$ & 0 \\
\hline $\mathrm{P}_{\mathrm{Kf}}{ }^{*} \cdot 10^{-9}\left[\mathrm{sm}^{3} \mathrm{~s}^{-1}\right]$ & 27 & $\underline{24.3}$ & $\underline{0}$ & 0 \\
\hline $\mathrm{P}_{\mathrm{Ks}} * 10^{-9}\left[\mathrm{sm}^{3} \mathrm{~s}^{-1}\right]$ & 2 & $\underline{1.8}$ & $\underline{0.6}$ & 0 \\
\hline $\mathrm{P}_{\mathrm{Lk}} * 10^{-11}\left[\mathrm{sm}^{3} \mathrm{~s}^{-1}\right]$ & 0.64 & $\underline{0.54}$ & $\underline{0.5}$ & 0.5 \\
\hline \hline
\end{tabular}

$R_{a x}$ (axoplasmic resistance); $R_{p n}$ (paranodal seal resistance); channel types and their maximum permeabilities: $P_{N a}$ (sodium), $P_{K f}$ (fast potassium), $P_{K s}$ (slow potassium), $P_{L k}$ (leak). The asterisk denotes an internodal quantity. The values showing the differences between the normal and each ALS case are underlined

When two equal-duration stimuli are used in pairs, the action potential in response to the second (testing) stimulus in the refractory period may be greater or lesser than that to the first (conditioning) stimulus and depends on the conditioning-test intervals. To obtain the time course of recovery of axonal excitability following a single threshold stimulus (recovery cycle), test stimuli of $1 \mathrm{~ms}$ duration are delivered at conditioning-test intervals of 2-100 ms after a threshold conditioning stimulus of $1 \mathrm{~ms}$ duration. The recovery cycles are calculated in the case of adaptation (i. e. in the case of intracellular current application delivered simultaneously to the centre of each internodal segment of the nerve fibre) and they are simulated by adding a long-duration suprathreshold depolarizing stimulus. This case closely approximates the effects of external surface stimulation with a large electrode and realizes a periodic kind of uniform fibre polarization.

\section{RESULTS}

The axonal excitability changes in the normal, demyelinated (systematically and focally) and ALS subtypes during $100 \mathrm{~ms}$ recovery cycles are illustrated in Fig. 

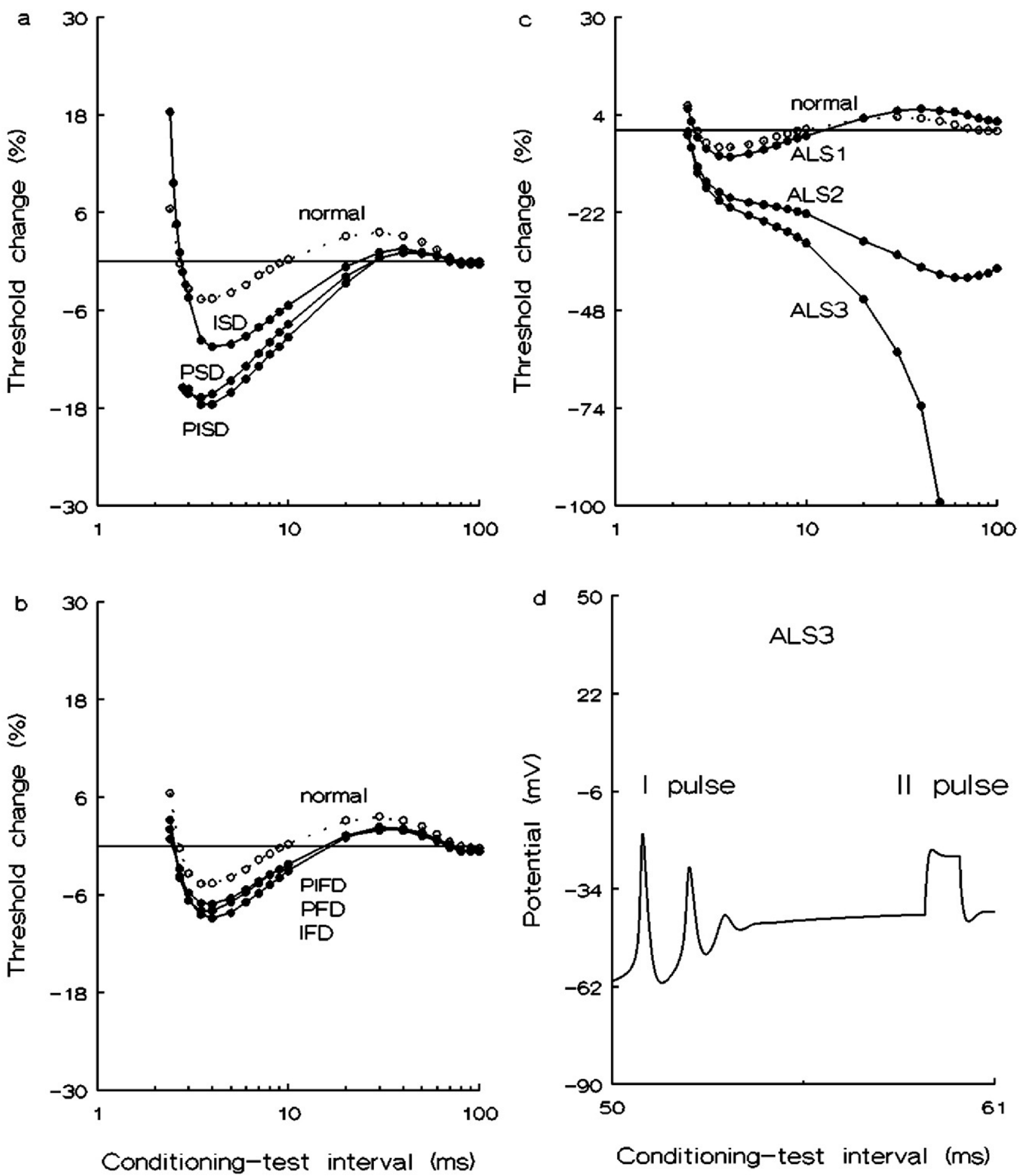

Fig. 2. Comparison between the recovery cycles in normal (dotted lines), mild ISD, PSD, PISD (a), severe IFD, PFD, PIFD (b), ALS1, ALS2, and ALS3 (c) cases of human motor nerve fibres. For all cases, the y-axis is defined as $100 x$ $\left(I_{\text {test }}-I_{\text {cond }}\right) / I_{\text {cond }}(\%)$, where $I_{\text {test }}(n A)$ and $I_{\text {cond }}(n A)$ are the threshold currents of the testing and conditioning pulses, respectively. Temporal distribution of the action potentials as a function of the conditioning-test interval in the ALS3 case (d). The conditioning and testing current stimuli are with duration of $1 \mathrm{~ms}$.

2a-c. The recovery cycles show that the excitability changes in the normal and ISD cases are similar (Fig. 2a). The axonal types are initially unexcitable, then excitable with a raised threshold and after about $2,5 \mathrm{~ms}$ they are superexcitable. In the unexcitable case, the axons are in the absolute refractory period during which they cannot generate second potential no matter how strong the testing depolarizing stimuli are. Then the axons are in the relative refractory period during which a stronger than conditioning stimulus is required to generate a second action potential. In 
the superexcitable case, the testing stimulus generating the second potential is less than the conditioning stimulus. Axonal superexcitability is usually followed by a late subexcitability. The ISD axon has greater refractoriness (the increase in threshold current during the relative refractory period), superexcitability and less late subexcitability than those for the normal axon. There is an increase in the refractoriness without an increase in the relative refractory period in this demyelinated axon. The recovery cycles show that the axonal excitability changes in the PSD and PISD cases are similar. Both axonal subtypes have abnormally greater superexcitabilities. In these cases compared to the normal one, the recovery cycles are without relative refractory periods and have only superexcitable and subexcitable periods.

The same investigations of axonal excitability changes during the $100 \mathrm{~ms}$ recovery cycles are repeated for the IFD, PFD and PIFD cases of the fibres and are illustrated in Fig. $2 b$. The recovery cycles show that the axonal excitability changes in all focally demyelinated cases are similar. The axons have slightly less refractoriness, greater superexcitability and less late subexcitability than those for the normal axon. However, the recovery cycles are near-overlap for the PFD and PIFD cases.

The excitability changes of human motor axons in the normal and ALS cases during the $100 \mathrm{~ms}$ recovery cycles are also illustrated (Fig. 2c). The recovery cycles show that the excitability changes of normal and abnormal axons are considerably different. The ALS1 axon has less refractoriness, greater superexcitability and late subexcitability than those for the normal one. For the ALS2 case, the superexcitability increases markedly and the axon remains superexcitable to the end of the investigated period. For the ALS3 case, the superexcitability increases rapidly and abnormalities are obtained in the recovery cycle beyond 50 ms conditioning-test intervals. In these cases, a block of each second stimulus applied to the internodal axolemma is achieved (Fig. 2d) as a result of axonal spontaneous activities caused by the first action potential.

\section{DISCUSSION}

Recovery cycle pattern obtained in the ISD, PSD and PISD cases is characteristic for patients with CMT1A, CIDP and CIDP subtypes, respectively $(9,15)$, whereas the recovery cycles obtained in the IFD, PFD and PIFD are typical for median motor nerves in AIDP patients $(7,10)$. The recovery cycle patterns in the simulated ALS1 and normal cases are similar. However, the refractoriness is less and the superexcitability is greater than those in the normal case and such results are also discussed by Mogyoros et al. (8) for ALS1 patients and normal controls.

The vastly greater superexcitabilities in the ALS2 case and the abnormalities obtained in the ALS3 case show how the recovery cycles are altered with the increase of the axonal ion channel dysfunction. The axonal superexcitability leads to blockage of each third stimulus (if it will be applied) in the case of ALS2 and to blockage of each second applied stimulus in the case of ALS3 as a result of repetitive firing caused by the previously applied stimulus. The superexcitability of the nodal and internodal axolemma based on the continuous activation and reactivation of the ionic (mainly $\mathrm{Na}^{+}$) channels in these compartments can be regarded as a prelude to neuron death and, probably, as a reason for motor neuron degeneration in this disease.

Our results show that recovery cycle abnormalities in the simulated demyelinating neuropathies are quite different from those in ALS because of the different fibre electrogenesis. The excitability abnormalities obtained in the simulated demyelinating neuropathies are based on the myelin sheath dysfunctions, whereas the excitability abnormalities obtained in the simulated subtypes ALS are based on the axonal dysfunctions along the length of human motor nerve fibres.

\section{REFERENCES}

1. Bostock, H., M. Baker. Evidence for two types of potassium channel in human motor axons in vivo.Brain Res, 462, 1988, No 2, 354-358.

2. Bostock, H., K. Cikurel, D. Burke. Threshold tracking techniques in the study the human peripheral nerve-- Muscle Nerve, 21, 1998, No 2, 137-158.

3. Bostock, H., M. K. Sharief, G. Reid, N. M. Murray. Axonal ion channel dysfunction in amyotrophic lateral sclerosis.- Brain, 118, 1995, No 1, 217-225.

4. Cappelen-Smith, C., S. Kuwabara, C. S.-Y Lin, I. Mogyoros, D. Burke. Membrane properties in chronic inflammatory demyelinating polyneuropathy.- Brain, 124, 2001, No 12, 2439-2447.

5. Kiernan, M. C., D. Burke, K. V. Andersen, H. Bostock. Multiple measures of axonal excitability: a new approach in clinical testing.- Muscle Nerve, 23, 2000, No 3, 399-409.

6. Kuwabara, S., H. Bostock, K. Ogawara, J. Y. Sung, K. Kanai, M. Mori, et al. The refractory period of transmission is impaired in axonal Guillain-Barrŭ syndrome.- Muscle Nerve, 28, 2003, No 6, 683-689.

7. Kuwabara, S., K. Ogawara, J. Y. Sung, M. Mori, K. Kanai, Hattori T, et al. Differences in membrane properties of axonal and demyelinating Guillain-Barrŭ syndromes.- Ann. Neurol., 52, 2002 , No 2, 180-187.

8. Mogyoros, I., M. C. Kiernan, D. Burke, H. Bostock. Strength-duration properties of sensory and motor axons in amyotrophic lateral sclerosis.Brain, 121, 1998, No 5, 851-859.

9. Nodera, H., H. Bostock, S. Kuwabara, T. Sakamoto, K. Asanuma, J. Y. Sung, et al. Nerve excitability properties in Charcot-Marie-Tooth disease type 1A.- Brain, 127, No 1, 2004, 203-211.

10. Nodera, H., R. Kaji. Nerve excitability testing and its clinical application to neuromuscular diseases.- Clin Neuphysiol, 117, 2006, No 9, 1902-1916. 
11. Stephanova, D. I. Myelin as longitudinal conductor: a multi-layered model of the myelinated human motor nerve fibre.- Biol. Cybern., 84, 2001, No 4, 301-308.

12. Stephanova, D. I. Excitability and potentials of human fibres in amyotrophic lateral sclerosis: model investigations.- In: Amyotrophic lateral sclerosis: new research. C. A. Murray, ed. New York, Nova Science Publishers Inc., 2006, 155-178.

13. Stephanova, D. I., M. S. Daskalova. Membrane property abnormalities in simulated cases of mild systematic and severe focal demyelinating neuropathies.- Eur. Biophys. J., 37, 2008, No 6, 183-195.

14. Stephanova, D. Comparison of membrane property abnormalities in simulated demyelinating neuropathies and neuronopathies.- Compt. Rend. Acad Bulg. Sci, 63, 2010, No 3, 435-442.

15. Sung, J. Y., S. Kuwabara, R. Kaji, K. Ogawara, M. Mori, K. Kanai, et al. Threshold electrotonus in chronic inflammatory demyelinating polyneuropathy: correlation with clinical profiles.- Muscle Nerve, 29, 2004, No 1, 28-37. 